\title{
Design and optimization for UAV-enabled two-way relaying system with
}

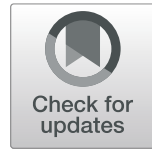
SWIPT

\author{
Jinlong Wang ${ }^{1}$, Gang Wang ${ }^{\text {* }}$ (D), Guanyi Chen ${ }^{1}$, Bo Li ${ }^{1}$, Ruofei Zhou ${ }^{1}$ and Ruoyu Zhang ${ }^{2}$
}

\begin{abstract}
In this paper, we investigate the resource allocation scheme for an unmanned-aerial-vehicle-enable (UAV-enabled) two-way relaying system with simultaneous wireless information and power transfer (SWIPT), where two user equipment exchange information with the help of UAV relay and harvest energy through power splitting (PS) scheme. Under the transmission power constraints at UEs and UAV relay, a non-convex intractable optimization problem is formulated which maximizes the sum retained energy of two UEs while satisfying the minimum signal-to-noise ratio requirement. We decouple the complicated beamforming and PS factor optimization problem into three solvable subproblems and propose an efficient alternating optimization scheme. Subsequently, in order to reduce the complexity, a robust scheme based on generalized singular value decomposition (GSVD) is designed. Finally, numerical results verify the robustness and effectiveness of the two proposed schemes.
\end{abstract}

Keywords: UAV-enabled, Resource allocation, SWIPT, MIMO, GSVD

\section{Introduction}

Recently, unmanned aerial vehicles (UAVs) have received dramatical attentions in wireless communication networks since they have the capability of flexible mobility [1-4]. Compared to the traditional wireless terminals, UAVs can be regarded as mobile relays or base stations. Therefore, UAV plays a critical role in the performance improvement and coverage extensiveness of the wireless communication network [5, 6]. In particular, UAV has huge potential in the intractable transmission environment, such as mountainous and emergency zones. Hence, the UAV which utilized as a relay has been widely investigated in the wireless network system. Specially, in [7], Song et al. investigated the instantaneous rate maximization problem for UAV-enabled decode-and-forward (DF) relay system by jointly optimizing the precoding matrix

\footnotetext{
*Correspondence: gwang51@hit.edu.cn

${ }^{1}$ Communication Research Center, Harbin Institute of Technology, 150001 Harbin, China

Full list of author information is available at the end of the article
}

and power allocation. Ji et al. [8] studied the secrecy performance analysis in a UAV DF relay system with energy harvesting. In [9], $\mathrm{HU}$ et al. considered the energy consumption minimization problem in UAV-assisted relay system, where UAV is regarded as a computation server for user equipment (UE) and forwards the result to the access point. Compared with one-way relay system, twoway relay system has higher spectrum efficiency. In [10], $\mathrm{Li}$ et al. provided a UAV-enable two-way relay system, where a set of UEs was considered. The UAV trajectory and the transmit power of all terminals were jointly optimized to maximize the sum rate of UEs.

In addition to the service coverage, the energy constraints of terminals also have a critical effect on the effectiveness and reliability of wireless networks [11-14]. In order to tackle this problem, simultaneous wireless information and power transfer (SWIPT) which can take advantage of the broadcast character of radio-frequency (RF) signal is adopted to enhance the performance of wireless communication networks [15-19]. In [20], two classical and feasible schemes, i.e., time switching (TS) and 
power splitting (PS) are proposed for SWIPT technique. The TS-based design switches over time between energy harvesting (EH) and information decoding (ID) processing, while the PS-based one splits the received RF signals into two power streams, one for ID and the other for EH. A multiple-input multiple-output (MIMO) relaying system with TS protocol was considered in [21], where the rate was maximized by designing the robust beamforming matrixes of transceivers under energy constraints. In [22], Wang et al. investigated a multi-antenna relay communication system with a TS-powered relay node. Furthermore, a robust precoding scheme that can maximize the sum-rate of the multiuser relay system has been proposed in [22]. Subsequently, in [23], a joint design of PS factor and the beamforming matrixes at the transceivers for MIMO two-way relay system based on maximizing the energy efficiency was introduced.

Inspired by the advantage of SWIPT, more and more researchers and scientists are attempting to adopt the SWIPT technique to UAV-enabled relay system for improving the system performance [24-26]. In [24], Wang et al. provided a UAV-enable non-orthogonal multiple access (NOMA) relay system with SWIPT, where the nonlinear energy harvesting model was considered. The PS factor and beamforming vectors were jointly optimized to maximize the rate of UEs while still guaranteeing security. Yin et al. [25] considered the throughput maximization problem in a UAV relay system with time-sharing mechanism, where the UAV relay is capable of SWIPT. In [26], the authors investigated a millimeter-wave UAV relay system with SWIPT to improve the secrecy performance, and the security rate of NOMA and orthogonal multiple access (OMA) were derived. However, the robust beamforming and SWIPT design for UAV-enabled relay system, where the UEs are capable of PS scheme, has not been well addressed.

\section{Methods}

In order to address the above practical issue, we investigate the UAV-enabled two-way relaying system with SWIPT. The main contributions are fourfold.

- We propose a UAV-enabled two-way relaying system with SWIPT. The system model distinguishes from the existing relaying systems, since the UEs are capable of PS protocol and multi-antennas are equipped at UEs.

- A novel optimization problem is formulated by maximizing the sum remained energy of two UEs subject to both UAV and UEs transmission power, while guaranteeing the minimum signal-to-noise ratio (SNR) requirement at UEs.

- We propose an alternating optimization (AO) scheme and a low-complexity scheme based on the generalized singular value decomposition (GSVD) for the formulated optimization problem.

- Simulation and numerical results are conducted to evaluate the robustness and effectiveness of two proposed schemes. Besides, the location ratio has been investigated to analyze the performance of the relay system.

The rest of the paper is outlined as follows. In Section 3, the UAV-enabled two-way relaying system with two PS UEs is described in detail. Section 4 concentrates on the design of the proposed $\mathrm{AO}$ scheme for maximizing the sum remained energy of two UEs. Section 5 provides the low-complexity scheme based on GSVD. In Section 6, numerical results are conducted to validate the performance of two proposed schemes. Finally, Section 7 gives the conclusion.

\section{System model and problem formulation}

\subsection{System model}

As shown in Fig. 1, we consider a UAV-enable two-way relay system where a UAV relay equipped with $N$ antennas helps two UEs to exchange information and two UEs, i.e., UE 1 and UE 2, are installed with the same antennas as UAV relay. Due to the barriers or buildings, there is no direct link between UE 1 and UE 2. For enhancing the reliability of the relay system, SWIPT technique is adopted at UEs, and the PS scheme is considered. To be specific, UEs split the received signal power into two parts, one for $\mathrm{EH}$ and the other for ID. We assume that all terminals know the channel state information (CSI) and the amplify-and-forward way is applied at UAV relay.

With the half-duplex relay, the communication period block $T$ is equally partitioned into two separate phases, i.e., multiple access (MAC) phase and broadcast (BC) phase. In the MAC phase, two UEs transmit their signals to UAV relay. The received signal at UAV relay can be written as

$$
\mathbf{y}_{\mathrm{R}}=\mathbf{H}_{1} \mathbf{W}_{1} \mathbf{x}_{1}+\mathbf{H}_{2} \mathbf{W}_{2} \mathbf{x}_{2}+\mathbf{n}_{R},
$$

where $\mathbf{H}_{i} \in \mathbb{C}^{N \times N}$ is the MIMO channel matrix from UE $i$ to UAV relay node, $\mathbf{W}_{i} \in \mathbb{C}^{N \times N}$ represents the beamforming matrix of UE $i$, which should be under the transmission power constraints $\operatorname{tr}\left(\mathbf{W}_{i} \mathbf{W}_{i}{ }^{H}\right)=P_{i}$ and $P_{i} \leqslant P_{i, \text { Max }}$, where $P_{i}$ and $P_{i, \operatorname{Max}}$ denote the realistic transmission power and the limitation of maximum transmission power at UE $i$, respectively. $\mathbf{x}_{i} \in \mathbb{C}^{N \times 1}$ is the original signal vector with $\mathbb{E}\left[\mathbf{x}_{i} \mathbf{x}_{i}^{H}\right]=\mathbf{I}_{N},(\cdot)^{H}$ is the conjugate transpose operation of one matrix, $\mathbf{n}_{R} \in \mathbb{C}^{N \times 1}$ represents the zero-mean additive white Gaussian noise (AWGN) at UAV relay with covariance matrix $\sigma_{R}^{2} \mathbf{I}_{N}$.

In the BC phase, UAV relay forwards its received signals with a beamforming matrix $\mathbf{W}_{R} \in \mathbb{C}^{N \times N}$, which should be under the transmission power constraints 


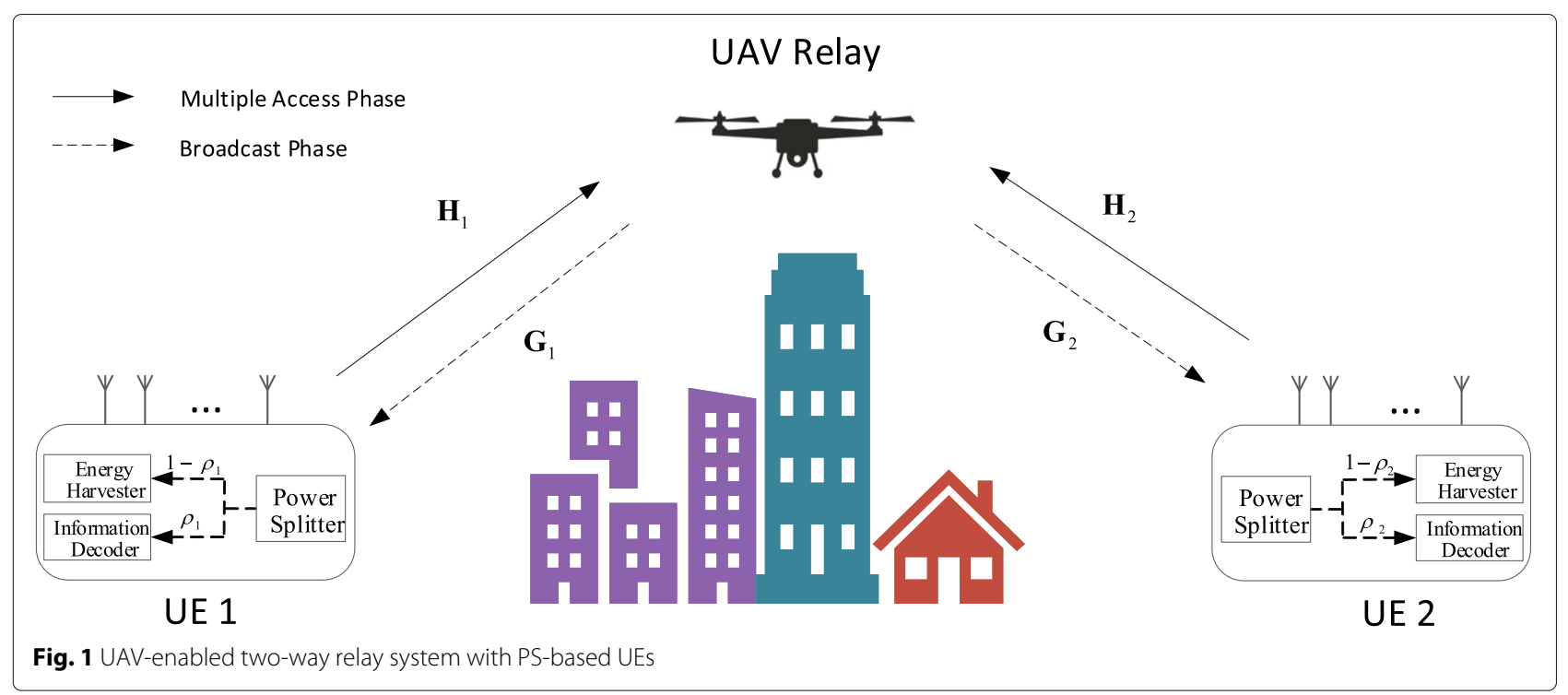

$\operatorname{tr}\left(\mathbf{W}_{R} \mathbf{R} \mathbf{W}_{R}^{H}\right)=P_{R}$ and $P_{R} \leqslant P_{R, \text { Max }}$, where $\mathbf{R}=$ $\mathbf{H}_{1} \mathbf{W}_{1} \mathbf{W}_{1}^{H} \mathbf{H}_{1}^{H}+\mathbf{H}_{2} \mathbf{W}_{2} \mathbf{W}_{2}^{H} \mathbf{H}_{2}^{H}+\sigma_{R}^{2} \mathbf{I}_{N}, P_{R}$ and $P_{R, \operatorname{Max}}$ are the realistic transmission power and the limitation of maximum transmission power at UAV relay, respectively. The signal processed by UAV relay is given by

$$
\mathbf{x}_{R}=\mathbf{W}_{R} \mathbf{y}_{R}=\mathbf{W}_{R}\left(\mathbf{H}_{1} \mathbf{W}_{1} \mathbf{x}_{1}+\mathbf{H}_{2} \mathbf{W}_{2} \mathbf{x}_{2}+\mathbf{n}_{R}\right) .
$$

The received RF signal at UE $i$ is split into two portions with a PS factor, i.e., $\rho_{i} \in(0,1) . \rho_{i}$ portion is used for ID, and $1-\rho_{i}$ portion is for $\mathrm{EH}$. In order to reduce the complexity of MIMO relay system, we assume that PS factors of all antennas at UE $i$ are the same. The ID signal at UE $i$ can be written as

$$
\begin{aligned}
\mathbf{y}_{i}=\sqrt{\rho_{i}}\left(\mathbf{G}_{i} \mathbf{W}_{R} \mathbf{H}_{i} \mathbf{W}_{i} \mathbf{x}_{i}+\mathbf{G}_{i} \mathbf{W}_{R} \mathbf{H}_{3-i} \mathbf{W}_{3-i} \mathbf{x}_{3-i}\right. \\
\left.+\mathbf{G}_{i} \mathbf{W}_{R} \mathbf{n}_{R}+\mathbf{n}_{i}\right)+\mathbf{n}_{i, z},
\end{aligned}
$$

where $\mathbf{n}_{i}$ indicates the zero-mean AWGN vector at UE $i$ with covariance matrix $\sigma_{i}^{2} \mathbf{I}_{N}, \mathbf{n}_{i, z}$ is the additional PS signal processing noise with covariance matrix $\sigma_{i, z}^{2} \mathbf{I}_{N} \cdot 3-i$ is used to denote the desire signal of paired user. As stated in [23], the self-interference term $\mathbf{G}_{i} \mathbf{W}_{R} \mathbf{H}_{i} \mathbf{W}_{i} \mathbf{x}_{i}$ can be integrally canceled. Therefore, the received signal at UE $i$ can be further obtained as

$\mathbf{y}_{i}=\sqrt{\rho_{i}}\left(\mathbf{G}_{i} \mathbf{W}_{R} \mathbf{H}_{3-i} \mathbf{W}_{3-i} \mathbf{x}_{3-i}+\mathbf{G}_{i} \mathbf{W}_{R} \mathbf{n}_{R}+\mathbf{n}_{i}\right)+\mathbf{n}_{i, z}$

According to (4), the received SNR at UE $i$ can be represented by

$$
S N R_{i}=\frac{\left\|\mathbf{G}_{i} \mathbf{W}_{R} \mathbf{H}_{3-i} \mathbf{W}_{3-i}\right\|^{2}}{\left\|\mathbf{G}_{i} \mathbf{W}_{R}\right\|^{2} \sigma_{R}^{2}+\left\|\sigma_{i}^{2} \mathbf{I}_{N}\right\|^{2}+\left\|\frac{\sigma_{i, z}^{2}}{\rho_{i}} \mathbf{I}_{N}\right\|^{2}} .
$$

The harvested energy at UE $i$ can be given by

$$
E_{i, e}^{H}=\frac{T}{2} \eta\left(1-\rho_{i}\right) \operatorname{tr}\left(\mathbf{G}_{i} \mathbf{W}_{R} \mathbf{R} \mathbf{W}_{R}^{H} \mathbf{G}_{i}^{H}+\sigma_{i}^{2} \mathbf{I}_{N}\right),
$$

where $\eta \in(0,1)$ is the energy conversion efficiency of UE $i$. It is worth pointing out that the self-interference term $\mathbf{G}_{i} \mathbf{W}_{R} \mathbf{H}_{i} \mathbf{W}_{i} \mathbf{x}_{i}$ can also be utilized for $\mathrm{EH}$.

\subsection{Problem formulation}

In this paper, we design the optimum beamforming matrixes at two UEs and UAV relay and two optimum PS ratios for SWIPT in two-way relay system to maximize the sum retained energy while satisfying sufficient SNR at two UEs and the transmit power constraint at each node. The retained energy of UE $i$ can be expressed as

$$
E_{i}^{R}=E_{i}^{I N I}+E_{i, e}^{H}-\frac{T}{2} P_{i}
$$

where $E_{i}^{I N I}$ denotes the initial energy at UE $i$. The sum retained energy of two UEs can be expressed as

$$
E_{\text {sum }}^{R}=\sum_{i=1}^{2} E_{i}^{R} .
$$

Based on (5) and (8), the optimization problem of sum retained energy is formulated as

$$
\begin{array}{ll} 
& \max _{\left(\mathbf{F}_{1}, \mathbf{F}_{2}, \mathbf{F}_{R}, \rho_{1}, \rho_{2}\right)} \mathrm{E}_{\text {sum }}^{R} \\
\text { s.t. } & S N R_{i} \geqslant \gamma_{i}, i \in\{1,2\}, \\
& \operatorname{Tr}\left(\mathbf{W}_{R} \mathbf{R} \mathbf{W}_{R}^{H}\right) \leqslant P_{R, \text { Max }}, \\
& \operatorname{Tr}\left(\mathbf{W}_{i} \mathbf{W}_{i}^{H}\right) \leqslant P_{i, \text { Max }}, i \in\{1,2\}, \\
& 0<\rho_{i}<1, i \in\{1,2\},
\end{array}
$$


where $\gamma_{i}$ indicates the SNR threshold of UE $i$, constraint (9b) represents the minimum SNR requirement of UE $i$, (9c) and (9d) denote the maximum transmission power of UAV relay and UE $i$, respectively, and (9e) is the PS factor constraint of UE $i$. Since the optimization problem is nonconvex, it is intractable to obtain the optimal solution of maximizing the sum retained energy.

\section{The proposed alternating optimization scheme}

In this section, we propose an $\mathrm{AO}$ scheme to convert the complicated optimization problem into three tractable subproblems. Firstly, we optimize the UE beamforming matrixes $\mathbf{W}_{1}$ and $\mathbf{W}_{2}$ with fixed $\mathbf{W}_{R}, \rho_{1}$ and $\rho_{2}$. Secondly, we try to obtain the optimal relay beamforming matrix $\mathbf{W}_{R}$ by assuming the other parameters are given. Finally, the optimal PS factors, i.e., $\rho_{1}$ and $\rho_{2}$, are investigated.

\subsection{Optimize two UE beamforming matrixes $\left(W_{1}\right.$ and $\left.W_{2}\right)$}

With the above analysis, we first design the optimal solution of $\mathbf{W}_{1}$ and $\mathbf{W}_{2}$ for maximizing the sum retained energy. Substituting $\mathbf{R}=\mathbf{H}_{1} \mathbf{W}_{1} \mathbf{W}_{1}^{H} \mathbf{H}_{1}{ }^{H}+$ $\mathbf{H}_{2} \mathbf{W}_{2} \mathbf{W}_{2}^{H} \mathbf{H}_{2}{ }^{H}+\sigma_{R}^{2} \mathbf{I}_{N}$ into (9), we have the objective function as

$$
\begin{aligned}
E_{\text {sum }}^{R}= & \frac{T}{2} \operatorname{tr}\left(\alpha_{1} \mathbf{T}_{1} \mathbf{W}_{1} \mathbf{W}_{1}^{H} \mathbf{T}_{1}^{H}+\alpha_{1} \mathbf{T}_{3} \mathbf{W}_{2} \mathbf{W}_{2}^{H} \mathbf{T}_{3}^{H}-\mathbf{W}_{1} \mathbf{W}_{1}^{H}\right) \\
& +\frac{T}{2} \operatorname{tr}\left(\alpha_{2} \mathbf{T}_{2} \mathbf{W}_{1} \mathbf{W}_{1}^{H} \mathbf{T}_{2}^{H}+\alpha_{2} \mathbf{T}_{4} \mathbf{W}_{2} \mathbf{W}_{2}^{H} \mathbf{T}_{4}^{H}-\mathbf{W}_{2} \mathbf{W}_{2}^{H}\right)+J,
\end{aligned}
$$

where $\alpha_{1}=\eta\left(1-\rho_{1}\right), \alpha_{2}=\eta\left(1-\rho_{2}\right), \mathbf{T}_{1}=$ $\mathbf{G}_{1} \mathbf{W}_{R} \mathbf{H}_{1}, \mathbf{T}_{2}=\mathbf{G}_{2} \mathbf{W}_{R} \mathbf{H}_{1}, \mathbf{T}_{3}=\mathbf{G}_{1} \mathbf{W}_{R} \mathbf{H}_{2}, \mathbf{T}_{4}=$ $\mathbf{G}_{2} \mathbf{W}_{R} \mathbf{H}_{2}, \mathbf{T}_{5}=\mathbf{G}_{1} \mathbf{W}_{R} \mathbf{W}_{R}^{H} \mathbf{G}_{1}^{H}, \mathbf{T}_{6}=\mathbf{G}_{2} \mathbf{W}_{R} \mathbf{W}_{R}^{H} \mathbf{G}_{2}^{H}$, and $J=\frac{T}{2} \alpha_{1} \operatorname{tr}\left(\sigma_{R}^{2} \mathbf{T}_{5}+\mathbf{I}_{N} \sigma_{1}^{2}\right)+\frac{T}{2} \alpha_{2} \operatorname{tr}\left(\sigma_{R}^{2} \mathbf{T}_{6}+\mathbf{I}_{N} \sigma_{2}^{2}\right)+$ $E_{1}^{I N I}+E_{2}^{I N I}$. Based on the trace property $\operatorname{tr}(\mathbf{A B})=$ $\operatorname{tr}(\mathbf{B A})$, the total retained energy can be rewritten as

$$
\begin{aligned}
E_{\text {sum }}^{R}= & \frac{T}{2} \operatorname{tr}\left(\Psi_{1} \mathbf{W}_{1} \mathbf{W}_{1}^{H}+\Psi_{2} \mathbf{W}_{1} \mathbf{W}_{1}^{H}-\mathbf{W}_{1} \mathbf{W}_{1}^{H}\right) \\
& +\frac{T}{2} \operatorname{tr}\left(\Psi_{3} \mathbf{W}_{2} \mathbf{W}_{2}^{H}+\Psi_{4} \mathbf{W}_{2} \mathbf{W}_{2}^{H}-\mathbf{W}_{2} \mathbf{W}_{2}^{H}\right)+J,
\end{aligned}
$$

where $\Psi_{1}=\alpha_{1} \mathbf{T}_{1}^{H} \mathbf{T}_{1}, \Psi_{2}=\alpha_{2} \mathbf{T}_{2}^{H} \mathbf{T}_{2}, \Psi_{3}=\alpha_{1} \mathbf{T}_{3}^{H} \mathbf{T}_{3}$, and $\Psi_{4}=\alpha_{2} \mathbf{T}_{4}^{H} \mathbf{T}_{4}$. With the trace property in [19], we yield

$$
\operatorname{tr}(\mathbf{A B C D})=\left(\operatorname{vec}\left(\mathbf{D}^{T}\right)\right)^{T}\left(\mathbf{C}^{T} \otimes \mathbf{A}\right) \operatorname{vec}(\mathbf{B}),
$$

where vec $(\cdot)$ is the matrix vectorization operator, $\otimes$ denotes the Kronecker product. From (12), we can reform the terms $\operatorname{tr}\left(\Psi_{1} \mathbf{W}_{1} \mathbf{W}_{1}^{H}\right)$ and $\operatorname{tr}\left(\mathbf{W}_{1} \mathbf{W}_{1}^{H}\right)$ into their following forms respectively

$$
\begin{aligned}
\operatorname{tr}\left(\Psi_{1} \mathbf{W}_{1} \mathbf{W}_{1}^{H}\right) & =\operatorname{tr}\left(\Psi_{1} \mathbf{W}_{1} \mathbf{I}_{N} \mathbf{W}_{1}^{H}\right) \\
& =\mathbf{w}_{1}^{H}\left(\mathbf{I}_{N} \otimes \Psi_{1}\right) \mathbf{w}_{1} \\
& =\operatorname{tr}\left(\left(\mathbf{I}_{N} \otimes \Psi_{1}\right) \mathbf{w}_{1} \mathbf{w}_{1}^{H}\right),
\end{aligned}
$$

and

$$
\operatorname{tr}\left(\mathbf{W}_{1} \mathbf{W}_{1}^{H}\right)=\mathbf{w}_{1}^{H} \mathbf{w}_{1}=\operatorname{tr}\left(\mathbf{w}_{1} \mathbf{w}_{1}^{H}\right),
$$

where $\mathbf{w}_{1}=\operatorname{vec}\left(\mathbf{W}_{1}\right)$. Similarly, we can convert other terms in (9). We define $\Phi_{i} \triangleq \mathbf{w}_{i} \mathbf{w}_{i}^{H}$, then the sum retained energy becomes

$$
\begin{aligned}
E_{\text {sum }}^{R}= & \frac{T}{2} \operatorname{tr}\left(\left(\left(\mathbf{I}_{N} \otimes \Psi_{1}\right)+\left(\mathbf{I}_{N} \otimes \Psi_{2}\right)-\mathbf{I}_{E}\right) \Phi_{1}\right) \\
& +\frac{T}{2} \operatorname{tr}\left(\left(\left(\mathbf{I}_{N} \otimes \Psi_{3}\right)+\left(\mathbf{I}_{N} \otimes \Psi_{4}\right)-\mathbf{I}_{E}\right) \Phi_{2}\right)+J,
\end{aligned}
$$

where $\mathbf{I}_{E} \in \mathbb{C}^{N^{2} \times N^{2}}$ is a unit matrix. With (5), we can transform the UEs SNR constraints into

$$
\begin{aligned}
& \operatorname{tr}\left(\left(\mathbf{I}_{N} \otimes \mathbf{T}_{3}^{H} \mathbf{T}_{3}\right) \Phi_{2}\right) \geqslant \gamma_{1} J_{1}, \\
& \operatorname{tr}\left(\left(\mathbf{I}_{N} \otimes \mathbf{T}_{2}^{H} \mathbf{T}_{2}\right) \Phi_{1}\right) \geqslant \gamma_{2} J_{2},
\end{aligned}
$$

where $J_{1}=\left\|\mathbf{G}_{1} \mathbf{F}_{R}\right\|^{2} \sigma_{R}^{2}+\sigma_{1}^{2}+\frac{\sigma_{1, z}^{2}}{\rho_{1}}$ and $J_{2}=\left\|\mathbf{G}_{2} \mathbf{F}_{R}\right\|^{2} \sigma_{R}^{2}+$ $\sigma_{2}^{2}+\frac{\sigma_{2, z}^{2}}{\rho_{2}}$. Similarly, the UAV relay transmission power constraint will become

$\operatorname{tr}\left(\left(\mathbf{I}_{N} \otimes \mathbf{T}_{5}\right) \Phi_{1}+\left(\mathbf{I}_{N} \otimes \mathbf{T}_{6}\right) \Phi_{2}+\sigma_{R}^{2} \mathbf{W}_{R} \mathbf{W}_{R}^{H}\right) \leqslant P_{R, \text { Max. }}$

Plugging (16), (17), and (18) back into (9), the original optimization problem can be reformulated as

$$
\begin{array}{ll}
\max _{\left(\Phi_{1}, \Phi_{2} \succcurlyeq \mathbf{0}\right)} & \frac{T}{2} \operatorname{tr}\left(\mathbf{Q}_{1} \Phi_{1}\right)+\frac{T}{2} \operatorname{tr}\left(\mathbf{Q}_{2} \Phi_{2}\right)+J \\
\text { s.t. } & \operatorname{tr}\left(\left(\mathbf{I}_{N} \otimes \mathbf{T}_{3}^{H} \mathbf{T}_{3}\right) \Phi_{2}\right) \geqslant \gamma_{1} J_{1}, \\
& \operatorname{tr}\left(\left(\mathbf{I}_{N} \otimes \mathbf{T}_{2}^{H} \mathbf{T}_{2}\right) \Phi_{1}\right) \geqslant \gamma_{2} J_{2}, \\
& \operatorname{tr}\left(\Phi_{i}\right) \leqslant P_{i, \text { Max }}, i \in\{1,2\}, \\
& \operatorname{tr}\left(\left(\mathbf{I}_{N} \otimes \mathbf{T}_{5}\right) \Phi_{1}+\left(\mathbf{I}_{N} \otimes \mathbf{T}_{6}\right) \Phi_{2}+\sigma_{R}^{2} \mathbf{W}_{R} \mathbf{W}_{R}^{H}\right) \\
& \leqslant P_{R, \text { Max }},
\end{array}
$$

where $\mathbf{Q}_{1}=\left(\mathbf{I}_{N} \otimes \Psi_{1}\right)+\left(\mathbf{I}_{N} \otimes \Psi_{2}\right)-\mathbf{I}_{E} \quad$ and $\mathbf{Q}_{2}=\left(\mathbf{I}_{N} \otimes \Psi_{3}\right)+\left(\mathbf{I}_{N} \otimes \Psi_{4}\right)-\mathbf{I}_{E}$. According to [27], the optimization problem (19) can be converted into a semidefinite programming (SDP) problem without a rank-one constraint. Due to the linearity of the objective function and the relaxed constraints, problem (18) which is convex can be solved by the classical optimization tools, e.g., CVX. Therefore, with the optimal solutions $\Phi_{1}^{*}$ and $\Phi_{2}^{*}$, we can obtain the optimal beamforming matrixes of UEs, i.e., $\mathbf{W}_{1}^{*}$ and $\mathbf{W}_{2}^{*}$.

\subsection{Optimize UAV relay beamforming $\left(\mathbf{W}_{R}\right)$}

Similarly, with given $\mathbf{W}_{1}, \mathbf{W}_{2}$ and two UE PS factors, i.e., $\rho_{1}, \rho_{2}$, the total retained energy of two UEs is equivalent to

$$
E_{\text {sum }}^{R}=\frac{T}{2} \operatorname{tr}\left(\tilde{\Psi}_{1} \mathbf{W}_{R} \mathbf{R} \mathbf{W}_{R}^{H}+\tilde{\Psi}_{2} \mathbf{W}_{R} \mathbf{R} \mathbf{W}_{R}^{H}\right)+\tilde{J},
$$


where $\tilde{\Psi}_{1}=\alpha_{1} \mathbf{G}_{1}^{H} \mathbf{G}_{1}, \tilde{\Psi}_{2}=\alpha_{2} \mathbf{G}_{2}^{H} \mathbf{G}_{2}$, and $\tilde{J}=\frac{T}{2} \operatorname{tr}$ $\left(\alpha_{1} \sigma_{1}^{2} \mathbf{I}_{N}+\alpha_{2} \sigma_{2}^{2} \mathbf{I}_{N}\right)+E_{1}^{I N I}+E_{2}^{I N I}-\frac{T}{2}\left(P_{1}+P_{2}\right)$. Based on (12), we have

$E_{\text {sum }}^{R}=\frac{T}{2} \operatorname{tr}\left(\left(\left(\mathbf{R}^{T} \otimes \tilde{\Psi}_{1}\right)+\left(\mathbf{R}^{T} \otimes \tilde{\Psi}_{2}\right)\right) \mathbf{w}_{R} \mathbf{w}_{R}^{H}\right)+\tilde{J}$,

where $\mathbf{w}_{R}=\operatorname{vec}\left(\mathbf{W}_{R}\right)$. Further, the constraints in (9) can be rewritten as

$$
\begin{aligned}
& \operatorname{tr}\left(\mathbf{K}_{1} \mathbf{w}_{R} \mathbf{w}_{R}^{H}\right) \geqslant \gamma_{1}\left(\sigma_{1}^{2}+\frac{\sigma_{1, z}^{2}}{\rho_{1}}\right), \\
& \operatorname{tr}\left(\mathbf{K}_{2} \mathbf{w}_{R} \mathbf{w}_{R}^{H}\right) \geqslant \gamma_{2}\left(\sigma_{2}^{2}+\frac{\sigma_{2, z}^{2}}{\rho_{2}}\right),
\end{aligned}
$$

and

$$
\operatorname{tr}\left(\left(\mathbf{R}^{T} \otimes \mathbf{I}_{N}\right) \mathbf{w}_{R} \mathbf{w}_{R}^{H}\right) \leqslant P_{R, \operatorname{Max}}
$$

where

$$
\mathbf{K}_{1}=\left(\left(\mathbf{H}_{2} \mathbf{F}_{2} \mathbf{F}_{2}^{H} \mathbf{H}_{2}^{H}\right)^{T} \otimes\left(\mathbf{G}_{1}^{H} \mathbf{G}_{1}\right)\right)-\gamma_{1} \sigma_{R}^{2}\left(\mathbf{I}_{N} \otimes\left(\mathbf{G}_{1}^{H} \mathbf{G}_{1}\right)\right),
$$

$\mathbf{K}_{2}=\left(\left(\mathbf{H}_{1} \mathbf{F}_{1} \mathbf{F}_{1}^{H} \mathbf{H}_{1}^{H}\right)^{T} \otimes\left(\mathbf{G}_{2}^{H} \mathbf{G}_{2}\right)\right)-\gamma_{2} \sigma_{R}^{2}\left(\mathbf{I}_{N} \otimes\left(\mathbf{G}_{2}^{H} \mathbf{G}_{2}\right)\right)$.

Here, we define $\Phi_{R} \triangleq \mathbf{w}_{R} \mathbf{w}_{R}^{H}$. Substituting (22), (23), and (24) back into (9), the original optimization problem can be reformulated as

$$
\begin{gathered}
\max _{\left(\Phi_{R} \succcurlyeq \mathbf{0}\right)} \frac{T}{2} \operatorname{tr}\left(\left(\left(\mathbf{R}^{T} \otimes \tilde{\Psi}_{1}\right)+\left(\mathbf{R}^{T} \otimes \tilde{\Psi}_{2}\right)\right) \Phi_{R}\right)+\tilde{J} \\
\text { s.t. } \operatorname{tr}\left(\mathbf{K}_{1} \Phi_{R}\right) \geqslant \gamma_{1}\left(\sigma_{1}^{2}+\frac{\sigma_{1, z}^{2}}{\rho_{1}}\right) \\
\operatorname{tr}\left(\mathbf{K}_{2} \Phi_{R}\right) \geqslant \gamma_{2}\left(\sigma_{2}^{2}+\frac{\sigma_{2, z}^{2}}{\rho_{2}}\right) \\
\operatorname{tr}\left(\left(\mathbf{R}^{T} \otimes \mathbf{I}_{N}\right) \Phi_{R}\right) \leqslant P_{R, \text { Max }} .
\end{gathered}
$$

Similar to the first subproblem, we can utilize SDP scheme to solve the problem (27). With the optimal solutions $\Phi_{R}^{*}$, we can get the optimal beamforming matrixes of UAV relay, i.e., $\mathbf{W}_{R}^{*}$.

\subsection{Optimize two PS factors $\left(\rho_{1}, \rho_{1}\right)$}

For given $\mathbf{W}_{1}, \mathbf{W}_{2}$, and $\mathbf{W}_{R}$, the objective function of maximizing the sum retained energy with $\rho_{1}$ and $\rho_{2}$ can be written as

$$
E_{\text {sum }}^{R}=\beta_{1}\left(1-\rho_{1}\right)+\beta_{2}\left(1-\rho_{2}\right)+\hat{J},
$$

where $\beta_{1}=\frac{T}{2} \eta \operatorname{tr}\left(\mathbf{G}_{1} \mathbf{W}_{R} \mathbf{R} \mathbf{W}_{R}^{H} \mathbf{G}_{1}^{H}+\sigma_{1}^{2} \mathbf{I}_{N}\right), \beta_{2}=\frac{T}{2} \eta \operatorname{tr}$ $\left(\mathbf{G}_{2} \mathbf{W}_{R} \mathbf{R} \mathbf{W}_{R}^{H} \mathbf{G}_{2}^{H}+\sigma_{2}^{2} \mathbf{I}_{N}\right)$, and $\hat{J}=E_{1}^{I N I}+E_{2}^{I N I}-\frac{T}{2}\left(P_{1}+P_{2}\right)$. According to the SNR constraints in (9), we obtain

$$
\begin{aligned}
& \rho_{1} \geqslant \frac{\sigma_{1, z}^{2} \gamma_{1}}{\mu_{1}}, \\
& \rho_{2} \geqslant \frac{\sigma_{2, z}^{2} \gamma_{2}}{\mu_{2}},
\end{aligned}
$$

where $\mu_{1}=\left\|\mathbf{G}_{1} \mathbf{W}_{R} \mathbf{H}_{2} \mathbf{W}_{2}\right\|^{2}-\gamma_{1} \sigma_{R}^{2}\left\|\mathbf{G}_{1} \mathbf{W}_{R}\right\|^{2}-\gamma_{1} \sigma_{1}^{2}$ and $\mu_{2}=\left\|\mathbf{G}_{2} \mathbf{W}_{R} \mathbf{H}_{1} \mathbf{F}_{1}\right\|^{2}-\gamma_{2} \sigma_{R}^{2}\left\|\mathbf{G}_{2} \mathbf{W}_{R}\right\|^{2}-\gamma_{2} \sigma_{2}^{2}$. Therefore, the original optimization problem can be changed as

$$
\begin{array}{ll}
\max _{\left(\rho_{1}, \rho_{2}\right)} & \beta_{1}\left(1-\rho_{1}\right)+\beta_{2}\left(1-\rho_{2}\right)+\hat{J} \\
\text { s.t. } & \rho_{1} \geqslant \frac{\sigma_{1, z}^{2} \gamma_{1}}{\mu_{1}}, \\
& \rho_{2} \geqslant \frac{\sigma_{2, z}^{2} \gamma_{2}}{\mu_{2}}, \\
& 0<\rho_{1}<1, \\
& 0<\rho_{2}<1 .
\end{array}
$$

With the known feasible region of the problem (31) and the relationship of the variates, the optimal closed-form expression of PS factors can be expressed as

$$
\begin{aligned}
& \rho_{1, \text { opt }}=\frac{\sigma_{1, z}^{2} \gamma_{1}}{\mu_{1}}, \\
& \rho_{2, \text { opt }}=\frac{\sigma_{2, z}^{2} \gamma_{2}}{\mu_{2}} .
\end{aligned}
$$

In summary, the proposed $\mathrm{AO}$ scheme for maximizing the sum remained energy can be summarized as Algorithm 1.

Algorithm 1 The proposed AO scheme for maximizing the sum retained energy

1: Initialization: Set $\rho_{1}=\rho_{2}=0.5, \mathbf{W}_{R}=$ $\sqrt{P_{R, \operatorname{Max}} / N \mathbf{I}_{N}}, \mathbf{W}_{1}=\sqrt{P_{1, \operatorname{Max}} / N \mathbf{I}_{N}}$ and $\mathbf{W}_{2}=$ $\sqrt{P_{2, \operatorname{Max}} / N} \mathbf{I}_{N}$.

\section{2: Repeat}

a) Update $\Phi_{1}$ and $\Phi_{2}$ by solving the convex problem (19) and to obtain $\mathbf{W}_{1}$ and $\mathbf{W}_{2}$;

b) Update $\Phi_{R}$ by solving the convex problem (27) and to obtain $\mathbf{W}_{R}$;

C) Update the optimal PS factors solution $\rho_{1, \text { opt }}$ and $\rho_{2, \text { opt }}$.

3: Until convergence.

Complexity Analysis: It is seen that the optimization problems (19) and (27) are two classical SDP problems which can be solved by utilizing the interior-point method (IPM). According to [18], the complexity of the proposed $\mathrm{AO}$ scheme for maximizing the sum retained energy is 
about $\mathcal{O}\left(M_{\text {Iter }} \cdot 3 N^{7}\right)$, where $M_{\text {Iter }}$ indicates the number of iterations.

\section{Low-complexity beamforming design based on GSVD technique}

The proposed AO scheme of UAV-enable two-way relay system in Section 4 gets great rate performance, whereas it has high computational complexity. To provide an outstanding balance between complexity and performance, a low-complexity scheme based on GSVD technique is presented in this section. Compared with the above AO scheme, the low-complexity GSVD scheme transforms the original optimization problem with matrix variables into the power allocation optimization problem with scalar variables. However, two schemes have the similar iteration process.

\subsection{Beamforming design based on GSVD}

By adopting GSVD technique onto the related MAC channel $\left[\mathbf{H}_{1}^{H}, \mathbf{H}_{2}^{H}\right]$, we can decompose $\mathbf{H}_{1}$ and $\mathbf{H}_{2}$ as

$$
\mathbf{H}_{1}=\mathbf{B}_{h} \Sigma_{1} \mathbf{A}_{h_{1}}^{H}, \quad \mathbf{H}_{2}=\mathbf{B}_{h} \Sigma_{2} \mathbf{A}_{h_{2}}^{H},
$$

where $\mathbf{B}_{h}$ denotes a full-rank complex matrix, $\Sigma_{1} \in \mathbb{C}^{N \times N}$ and $\Sigma_{2} \in \mathbb{C}^{N \times N}$ are two diagonal matrixes, $\mathbf{A}_{h_{1}} \in \mathbb{C}^{N \times N}$ and $\mathbf{A}_{h_{2}} \in \mathbb{C}^{N \times N}$ represent two unitary matrixes. After processing the MAC channel, we apply SVD technique to decompose the BC channel $\mathbf{G}_{B C}=\left[\mathbf{G}_{1}^{T}, \mathbf{G}_{2}^{T}\right]^{T}$ as

$$
\mathbf{G}_{B C}=\mathbf{B}_{g} \Sigma_{g} \mathbf{A}_{g}^{H},
$$

where $\mathbf{B}_{g} \in \mathbb{C}^{2 N \times 2 N}$ and $\mathbf{A}_{g} \in \mathbb{C}^{N \times N}$ denotes two unitary matrixes, and $\Sigma_{g} \in \mathbb{C}^{N \times N}$ is a diagonal matrix with nonegative real values arranged into decreasing order. Based on (35), $\mathbf{G}_{1}$ and $\mathbf{G}_{2}$ are decomposed as [17, 28]

$$
\mathbf{G}_{1}=\mathbf{B}_{g_{1}} \Sigma_{g} \mathbf{A}_{g}^{H}, \quad \mathbf{G}_{2}=\mathbf{B}_{g_{2}} \Sigma_{g} \mathbf{A}_{g}^{H},
$$

where $\mathbf{B}_{g_{1}}=\mathbf{B}_{g}(1: N, 1: N)$ and $\mathbf{B}_{g_{2}}=$ $\mathbf{B}_{g}(N+1: 2 N, 1: N)$. It is worth noting that $\mathbf{B}_{g_{i}}$ is not a unitary matrix. According to the above decompositions of MAC and $\mathrm{BC}$ channel matrixes, the beamforming structures of UE $i$ and UAV relay can be proposed as

$$
\mathbf{W}_{i}=\mathbf{A}_{h_{i}} \Lambda_{i}, \quad \mathbf{W}_{R}=\mathbf{A}_{g} \Lambda_{R} \mathbf{B}_{h}^{-1},
$$

where $\Lambda_{i} \in \mathbb{C}^{N \times N}$ and $\Lambda_{R} \in \mathbb{C}^{N \times N}$ are two diagonal matrixes standing for the power allocation of UE $i$ and UAV relay, respectively. Plugging (34), (36), and (37) into (4), the receive signal at UE $i$ can be written as

$$
\begin{aligned}
\mathbf{y}_{i}= & \sqrt{\rho_{i}}\left(\mathbf{B}_{g_{i}} \Sigma_{g} \Lambda_{R} \Sigma_{3-i} \Lambda_{3-i} \mathbf{x}_{3-i}+\mathbf{B}_{g_{i}} \Sigma_{g} \Lambda_{R} \mathbf{B}_{h}^{-1} \mathbf{n}_{R}+\mathbf{n}_{i}\right) \\
& +\mathbf{n}_{i, z} .
\end{aligned}
$$

After using the zero-forcing operation for the received signal at $\mathrm{UE} i$, i.e., $\mathbf{B}_{g_{i}}^{-1} \mathbf{y}_{i}$, we have

$\tilde{\mathbf{y}}_{i}=\sqrt{\rho_{i}}\left(\Sigma_{g} \Lambda_{R} \Sigma_{3-i} \Lambda_{3-i} \mathbf{x}_{3-i}+\Sigma_{g} \Lambda_{R} \tilde{\mathbf{n}}_{R}+\tilde{\mathbf{n}}_{i}\right)+\tilde{\mathbf{n}}_{i, z}$,

where $\tilde{\mathbf{y}}_{i}=\mathbf{B}_{g_{i}}^{-1} \mathbf{y}_{i}, \tilde{\mathbf{n}}_{R}=\mathbf{B}_{h}^{-1} \mathbf{n}_{R}, \tilde{\mathbf{n}}_{i}=\mathbf{B}_{g_{i}}^{-1} \mathbf{n}_{i}$, and $\tilde{\mathbf{n}}_{i, z}=$ $\mathbf{B}_{g_{i}}^{-1} \mathbf{n}_{i, z}$. Substituting (37) back into (7), we can transform the retained energy $E_{i}^{R}$ into its equivalent form as

$$
\begin{aligned}
E_{i}^{R}= & \frac{T}{2} \eta\left(1-\rho_{i}\right) \operatorname{tr}\left(\Psi_{g_{i}} \Sigma_{g} \Lambda_{R}\left(\Pi+\sigma_{R}^{2} \Psi_{h}\right) \Lambda_{R}^{H} \Sigma_{g}^{H}+\sigma_{i}^{2} \mathbf{I}_{N}\right) \\
& +E_{i}^{I N I}-\frac{T}{2} \operatorname{tr}\left(\Lambda_{i} \Lambda_{i}^{H}\right),
\end{aligned}
$$

where $\Psi_{g_{i}}=\mathbf{B}_{g_{i}}^{H} \mathbf{B}_{g_{i}}, \Psi_{h}=\left(\mathbf{B}_{h}^{H} \mathbf{B}_{h}\right)^{-1}$, and $\Pi=$ $\Sigma_{1} \Lambda_{1} \Lambda_{1}^{H} \Sigma_{1}^{H}+\Sigma_{2} \Lambda_{2} \Lambda_{2}^{H} \Sigma_{2}^{H}$. With the transformation of the optimization constraints, the problem (9) can be reformulated as

$$
\begin{array}{ll} 
& \max _{\left(\Lambda_{1}, \Lambda_{2}, \Lambda_{R}, \rho_{1}, \rho_{2}\right)} E_{1}^{R}+E_{2}^{R} \\
\text { s.t. } & \frac{\operatorname{tr}\left(\Sigma_{g} \Lambda_{R} \Sigma_{3-i} \Lambda_{3-i} \Lambda_{3-i}^{H} \Sigma_{3-i}^{H} \Lambda_{R}^{H} \Sigma_{g}^{H}\right)}{\operatorname{tr}\left(\Lambda_{R}^{H} \Sigma_{g}^{H} \Sigma_{g} \Lambda_{R} \Psi_{h}\right) \sigma_{R}^{2}+\operatorname{tr}\left(\Psi_{g_{i}}^{-1}\right) \sigma_{i}^{2}+\operatorname{tr}\left(\Psi_{g_{i}}^{-1}\right) \frac{\sigma_{i z}^{2}}{\rho_{i}}} \geqslant \gamma_{i}, i \in\{1,2\}, \\
& \operatorname{tr}\left(\Lambda_{R}\left(\Pi+\Psi_{h} \sigma_{R}^{2}\right) \Lambda_{R}^{H}\right) \leqslant P_{R, M a x}, \\
& \operatorname{tr}\left(\Lambda_{i} \Lambda_{i}^{H}\right) \leqslant P_{i, \operatorname{Max}}, i \in\{1,2\}, \\
& 0<\rho_{i}<1, i \in\{1,2\} .
\end{array}
$$

Obviously, the trace matrixes of the retained energy $E_{i}^{R}$ and two constraints in (41) are not diagonal. According to Property 1 in [17], we can achieve a lower bound on the retained energy to simplify the optimization problem (41). A lower bound of $E_{i}^{R}$ is expressed as

$$
\begin{aligned}
E_{i}^{R} \geqslant \widehat{E}_{i}^{R}= & \frac{T}{2} \eta\left(1-\rho_{i}\right) \operatorname{tr}\left(\Lambda_{g_{i}} \Sigma_{g} \Lambda_{R}\left(\Pi+\sigma_{R}^{2} \Lambda_{h}\right) \Lambda_{R}^{H} \Sigma_{g}^{H}+\sigma_{i}^{2} \mathbf{I}_{N}\right) \\
& +E_{i}^{I N I}-\frac{T}{2} \operatorname{tr}\left(\Lambda_{i} \Lambda_{i}^{H}\right),
\end{aligned}
$$

where $\Lambda_{g_{i}}$ and $\Lambda_{h}$ are two diagonal matrixes that consist of $N$ diagonal elements of $\Psi_{g_{i}}$ and $\Psi_{h}$. For arbitrary positive semi-definite matrixes $\mathbf{A}$ and $\mathbf{X}$, there are $\operatorname{tr}(\mathbf{A X}) \geqslant \operatorname{tr}\left(\mathbf{A} \Lambda_{X}\right)$ in [17] and $\operatorname{tr}\left(\mathbf{X}^{-1}\right) \geqslant \sum_{i}[\mathbf{X}(i, i)]^{-1}$ in [26], where $\Lambda_{X}$ is a diagonal matrix that comprises the diagonal elements of $\mathbf{X}$. Hence, we can obtain the upper bound of SNR constraint (41a) and the lower bound of UAV relay transmission power constraint (41b) respectively as 


$$
\begin{gathered}
\frac{\operatorname{tr}\left(\Sigma_{g} \Lambda_{R} \Sigma_{3-i} \Lambda_{3-i} \Lambda_{3-i}^{H} \Sigma_{3-i}^{H} \Lambda_{R}^{H} \Sigma_{g}^{H}\right)}{\operatorname{tr}\left(\Lambda_{R}^{H} \Sigma_{g}^{H} \Sigma_{g} \Lambda_{R} \Psi_{h}\right) \sigma_{R}^{2}+\operatorname{tr}\left(\Psi_{g_{i}}^{-1}\right) \sigma_{i}^{2}+\operatorname{tr}\left(\Psi_{g_{i}}^{-1}\right) \frac{\sigma_{i, z}^{2}}{\rho_{i}}} \\
\leqslant \frac{\operatorname{tr}\left(\Sigma_{g} \Lambda_{R} \Sigma_{3-i} \Lambda_{3-i} \Lambda_{3-i}^{H} \Sigma_{3-i}^{H} \Lambda_{R}^{H} \Sigma_{g}^{H}\right)}{\operatorname{tr}\left(\Lambda_{R}^{H} \Sigma_{g}^{H} \Sigma_{g} \Lambda_{R} \Lambda_{h}\right) \sigma_{R}^{2}+\operatorname{tr}\left(\Lambda_{g_{i}}^{-1}\right) \sigma_{i}^{2}+\operatorname{tr}\left(\Lambda_{g_{i}}^{-1}\right) \frac{\sigma_{i, z}^{2}}{\rho_{i}}},
\end{gathered}
$$

$$
\operatorname{tr}\left(\Lambda_{R}\left(\Pi+\Psi_{h} \sigma_{R}^{2}\right) \Lambda_{R}^{H}\right) \geqslant \operatorname{tr}\left(\Lambda_{R}\left(\Pi+\Lambda_{h} \sigma_{R}^{2}\right) \Lambda_{R}^{H}\right)
$$

With (42), (43), and (44), we can reformulate the problem (41) as

$$
\begin{aligned}
& \max _{\left(\Lambda_{1}, \Lambda_{2}, \Lambda_{R}, \rho_{1}, \rho_{2}\right)} \stackrel{\overbrace{}^{R}}{E_{1}}+\stackrel{\overbrace{E_{2}}^{R}}{ } \\
& \text { s.t. } \frac{\operatorname{tr}\left(\Sigma_{g} \Lambda_{R} \Sigma_{3-i} \Lambda_{3-i} \Lambda_{3-i}^{H} \Sigma_{3-i}^{H} \Lambda_{R}^{H} \Sigma_{g}^{H}\right)}{\operatorname{tr}\left(\Lambda_{R}^{H} \Sigma_{g}^{H} \Sigma_{g} \Lambda_{R} \Lambda_{h}\right) \sigma_{R}^{2}+\operatorname{tr}\left(\Lambda_{g_{i}}^{-1}\right) \sigma_{i}^{2}+\operatorname{tr}\left(\Lambda_{g_{i}}^{-1}\right) \frac{\sigma_{i, z}^{2}}{\rho_{i}}} \\
& \geqslant \gamma_{i}, i \in\{1,2\} \text {, } \\
& \operatorname{tr}\left(\Lambda_{R}\left(\Pi+\Lambda_{h} \sigma_{R}^{2}\right) \Lambda_{R}^{H}\right) \leqslant P_{R, \text { Max }}, \\
& \operatorname{tr}\left(\Lambda_{i} \Lambda_{i}^{H}\right) \leqslant P_{i, \operatorname{Max}}, i \in\{1,2\} \text {, } \\
& 0<\rho_{i}<1, i \in\{1,2\} \text {. }
\end{aligned}
$$

\subsection{Optimize UE power allocation}

In the following, we adopt the AO-based optimization algorithm to solve the intractable and non-convex problem (45). For given $\Lambda_{R}, \rho_{1}$, and $\rho_{2}$, the objective function of the sum retained energy can be rewritten as

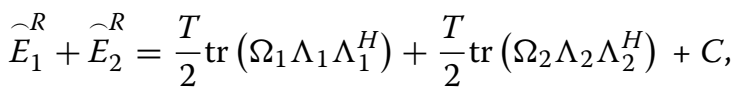

where

$\Omega_{1}=\left(\Sigma_{g} \Lambda_{R} \Sigma_{1}\right)^{H}\left(\alpha_{1} \Lambda_{g_{1}}+\alpha_{2} \Lambda_{g_{2}}\right)\left(\Sigma_{g} \Lambda_{R} \Sigma_{1}\right)-\mathbf{I}_{N}$,

$\Omega_{2}=\left(\Sigma_{g} \Lambda_{R} \Sigma_{2}\right)^{H}\left(\alpha_{1} \Lambda_{g_{1}}+\alpha_{2} \Lambda_{g_{2}}\right)\left(\Sigma_{g} \Lambda_{R} \Sigma_{2}\right)-\mathbf{I}_{N}$

and

$$
\begin{aligned}
C= & \frac{T}{2} \alpha_{1} \operatorname{tr}\left(\Lambda_{g_{1}} \Sigma_{g} \Lambda_{R} \Lambda_{h} \Lambda_{R}^{H} \Sigma_{g}^{H}+\sigma_{1}^{2} \mathbf{I}_{N}\right) \\
& +\frac{T}{2} \alpha_{2} \operatorname{tr}\left(\Lambda_{g_{2}} \Sigma_{g} \Lambda_{R} \Lambda_{h} \Lambda_{R}^{H} \Sigma_{g}^{H}+\sigma_{2}^{2} \mathbf{I}_{N}\right) \\
& +E_{1}^{I N I}+E_{2}^{I N I} .
\end{aligned}
$$

Define $\lambda_{i, n}, \lambda_{R, n}, \lambda_{h_{i}, n}, \lambda_{g, n}, \lambda_{\Psi_{h}, n}$, and $\lambda_{\Psi_{g_{i}, n}}$ as the $n$th diagonal entries of $\Lambda_{i}, \Lambda_{R}, \Sigma_{i}, \Sigma_{g}, \Lambda_{h}$, and $\Lambda_{g_{i}}$, respectively. Following this definitions, the sum retained energy can be transformed in a scalar form as

$\stackrel{\overbrace{E_{1}}^{R}}{\stackrel{E^{R}}{R}}=\frac{T}{2}\left(\sum_{n=1}^{N} \lambda_{\Omega_{1}, n} \lambda_{1, n}^{2}+\sum_{n=1}^{N} \lambda_{\Omega_{2}, n} \lambda_{2, n}^{2}\right)+C$, where $\lambda_{\Omega_{i}, n}$ denotes the $n$th diagonal entries of $\Omega_{i}$. With (45) and (50), the original optimization problem (9) with matrix variables is reformulated as the following power allocation problem with scalar variables

$$
\begin{aligned}
\max _{\left(\lambda_{1}, \lambda_{2}\right)} & \frac{T}{2}\left(\sum_{n=1}^{N} \lambda_{\Omega_{1}, n} \lambda_{1, n}^{2}+\sum_{n=1}^{N} \lambda_{\Omega_{2}, n} \lambda_{2, n}^{2}\right)+C \\
\text { s.t. } & \frac{\sum_{n=1}^{N} \lambda_{g, n}^{2} \lambda_{R, n}^{2} \lambda_{h_{3-i}, n}^{2} \lambda_{3-i, n}^{2}}{\sum_{n=1}^{N} \lambda_{g, n}^{2} \lambda_{R, n}^{2} \lambda \Psi_{h, n} \sigma_{R}^{2}+\sum_{n=1}^{N} \frac{\sigma_{i}^{2}}{\lambda \Psi_{g_{i}, n}}+\sum_{n=1}^{N} \frac{\sigma_{i, z}^{2}}{\lambda_{g_{i}, n} \rho_{i}}} \geqslant \gamma_{i}, i \in\{1,2\}, \\
& \sum_{n=1}^{N} \lambda_{R, n}^{2}\left(\lambda_{h_{1}, n}^{2} \lambda_{1, n}^{2}+\lambda_{h_{2}, n}^{2} \lambda_{2, n}^{2}+\lambda \Psi_{h, n} \sigma_{R}^{2}\right) \leqslant P_{R, \text { Max }} \\
& \sum_{n=1}^{N} \lambda_{i, n}^{2} \leqslant P_{i, \operatorname{Max}}, i \in\{1,2\}
\end{aligned}
$$

where $\lambda_{i}=\left[\lambda_{i, 1}^{2}, \cdots, \lambda_{i, N}^{2}\right]^{T}$ indicates the allocation power of the subchannels at UE $i$. Since the linearity of the objective function with the terms of $\lambda_{1}$ and $\lambda_{2}$, the optimization problem (51) is convex and can be readily solved through the classical optimization scheme, i.e., CVX tool.

\subsection{Optimize UAV relay power allocation}

Similarly, for given $\Lambda_{1}, \Lambda_{2}, \rho_{1}$, and $\rho_{2}$, we have the sum retained energy function with UAV relay power allocation matrix $\Lambda_{R}$ as

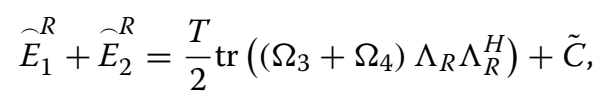

where

$$
\begin{aligned}
& \Omega_{3}=\alpha_{1} \Sigma_{g}^{H} \Lambda_{g_{1}} \Sigma_{g}\left(\Pi+\sigma_{R}^{2} \Lambda_{h}\right), \\
& \Omega_{4}=\alpha_{2} \Sigma_{g}^{H} \Lambda_{g_{2}} \Sigma_{g}\left(\Pi+\sigma_{R}^{2} \Lambda_{h}\right)
\end{aligned}
$$

and

$$
\begin{aligned}
\tilde{C}= & \frac{T}{2} \operatorname{tr}\left(\alpha_{1} \sigma_{1}^{2} \mathbf{I}_{N}+\alpha_{2} \sigma_{2}^{2} \mathbf{I}_{N}\right)+E_{1}^{I N I}+E_{2}^{I N I} \\
& -\frac{T}{2} \operatorname{tr}\left(\Lambda_{1} \Lambda_{1}^{H}+\Lambda_{2} \Lambda_{2}^{H}\right) .
\end{aligned}
$$

According to (50), (52) can be transformed in a scalar form as

$$
\stackrel{\overbrace{E_{1}}^{R}}{\overbrace{E_{2}}^{R}}=\frac{T}{2} \sum_{n=1}^{N}\left(\left(\lambda_{\Omega_{3}, n}+\lambda_{\Omega_{4}, n}\right) \lambda_{R, n}^{2}\right)+\tilde{C},
$$

where $\lambda_{\Omega_{3}, n}$ and $\lambda_{\Omega_{4}, n}$ strand for the $n$th diagonal entries of $\Omega_{3}$ and $\Omega_{4}$, respectively. Combining (45) with (56), the original optimization problem in (9) can be restated with scalar variables as 


$$
\begin{gathered}
\max _{\left(\lambda_{R}\right)} \frac{T}{2} \sum_{n=1}^{N}\left(\left(\lambda_{\Omega_{3}, n}+\lambda_{\Omega_{4}, n}\right) \lambda_{R, n}^{2}\right)+\tilde{C} \\
\text { s.t. } \sum_{n=1}^{N}\left(\left(\lambda_{g, n}^{2} \lambda_{h_{3-i}, n}^{2} \lambda_{3-i, n}^{2}-\gamma_{i} \lambda_{g, n}^{2} \lambda_{\Psi_{h}, n} \sigma_{R}^{2}\right) \lambda_{R, n}^{2}\right) \\
\geqslant \gamma_{i}\left(\sum_{n=1}^{N} \frac{\sigma_{i}^{2}}{\lambda \Psi_{g_{i}, n}}+\sum_{n=1}^{N} \frac{\sigma_{i, z}^{2}}{\lambda \Psi_{g_{i}, n} \rho_{i}}\right), i \in\{1,2\}
\end{gathered}
$$

$$
\sum_{n=1}^{N}\left(\left(\lambda_{h_{1}, n}^{2} \lambda_{1, n}^{2}+\lambda_{h_{2}, n}^{2} \lambda_{2, n}^{2}+\lambda_{\Psi_{h, n}} \sigma_{R}^{2}\right) \lambda_{R, n}^{2}\right) \leqslant P_{R, \operatorname{Max}}
$$

where $\lambda_{R}=\left[\lambda_{R, 1}^{2}, \cdots, \lambda_{R, N}^{2}\right]^{T}$ is the allocation power of the subchannels at UAV relay. Due to the linearity of the objective function with the term of $\lambda_{R}$, the problem (57) is also convex and can be efficiently solved by CVX tool.

\subsection{Optimize PS factors}

For given $\Lambda_{1}, \Lambda_{2}$, and $\Lambda_{R}$, the objective function of maximizing the sum retained energy with $\rho_{1}$ and $\rho_{2}$ can be written as

$$
\stackrel{\overbrace{}^{R}}{E_{1}}+\stackrel{\overbrace{}^{R}}{2}=\hat{\beta_{1}}\left(1-\rho_{1}\right)+\hat{\beta}_{2}\left(1-\rho_{2}\right)+\hat{C},
$$

where

$\hat{\beta_{1}}=\frac{T}{2} \eta \operatorname{tr}\left(\Lambda_{g_{1}} \Sigma_{g} \Lambda_{R}\left(\Pi+\Lambda_{h} \sigma_{R}^{2}\right) \Lambda_{R}^{H} \Sigma_{g}^{H}+\sigma_{1}^{2} \mathbf{I}_{N}\right)$,

$\hat{\beta_{2}}=\frac{T}{2} \eta \operatorname{tr}\left(\Lambda_{g_{2}} \Sigma_{g} \Lambda_{R}\left(\Pi+\Lambda_{h} \sigma_{R}^{2}\right) \Lambda_{R}^{H} \Sigma_{g}^{H}+\sigma_{2}^{2} \mathbf{I}_{N}\right)$

and

$$
\hat{C}=E_{1}^{I N I}+E_{2}^{I N I}-\frac{T}{2} \operatorname{tr}\left(\Lambda_{1} \Lambda_{1}^{H}+\Lambda_{2} \Lambda_{2}^{H}\right) .
$$

Based on the SNR constraint in (45), we have

$$
\rho_{i} \geqslant \frac{\gamma_{i} \operatorname{tr}\left(\Lambda_{g_{i}}^{-1}\right) \sigma_{i, z}^{2}}{\hat{\mu}_{i}},
$$

where

$$
\begin{aligned}
\hat{\mu}_{i}= & \operatorname{tr}\left(\Sigma_{g} \Lambda_{R} \Sigma_{3-i} \Lambda_{3-i} \Lambda_{3-i}^{H} \Sigma_{3-i}^{H} \Lambda_{R}^{H} \Sigma_{g}^{H}\right) \\
& -\gamma_{i}\left(\operatorname{tr}\left(\Lambda_{R}^{H} \Sigma_{g}^{H} \Sigma_{g} \Lambda_{R} \Lambda_{h}\right) \sigma_{R}^{2}+\operatorname{tr}\left(\Lambda_{g_{i}}^{-1}\right) \sigma_{i}^{2}\right) .
\end{aligned}
$$

Combining (58) and (62), the original optimization problem can be reformulated as

$$
\begin{aligned}
& \max _{\left(\rho_{1}, \rho_{2}\right)} \hat{\beta}_{1}\left(1-\rho_{1}\right)+\hat{\beta}_{2}\left(1-\rho_{2}\right)+\hat{C} \\
& \text { s.t. } \quad \rho_{i} \geqslant \frac{\gamma_{i} \operatorname{tr}\left(\Lambda_{g_{i}}^{-1}\right) \sigma_{i, z}^{2}}{\hat{\mu}_{i}}, i \in\{1,2\}, \\
& 0<\rho_{i}<1, i \in\{1,2\} .
\end{aligned}
$$

From the relationship of variates in (64), the optimal closed-form expression of PS factors can be expressed as

$$
\rho_{i, \text { opt }}=\frac{\gamma_{i} \operatorname{tr}\left(\Lambda_{g_{i}}^{-1}\right) \sigma_{i, z}^{2}}{\hat{\mu}_{i}}, i \in\{1,2\} .
$$

In summary, the proposed low-complexity $\mathrm{AO}$ scheme based on GSVD for maximizing the sum remained energy can be summarized as Algorithm 2.

$\overline{\text { Algorithm } 2 \text { Low-complexity beamforming design for }}$ maximizing the sum retained energy

1: Initialization: Set $\rho_{1}=\rho_{2}=0.5$ and $\Lambda_{1}=\Lambda_{2}=$ $\Lambda_{R}=\mathbf{I}_{N}$.

2: Adopt GSVD and SVD techniques to decompose $\left[\mathbf{H}_{1}^{H}, \mathbf{H}_{2}^{H}\right]$ and $\left[\mathbf{G}_{1}^{T}, \mathbf{G}_{2}^{T}\right]^{T}$, respectively, and then obtain $\Sigma_{1}, \Sigma_{2}, \Sigma_{g}, \Lambda_{h}, \Lambda_{g_{1}}$ and $\Lambda_{g_{2}}$.

3: Repeat

a) Update the transmission power allocation of UEs $\Lambda_{1}$ and $\Lambda_{2}$ by solving the convex problem (51);

b) Update the transmission power allocation of UAV relay $\Lambda_{R}$ by solving the convex problem (57); c) Update the optimal PS factors solution $\rho_{1, \text { opt }}$ and $\rho_{1, \text { opt }}$.

\section{4: Until convergence}

5: Plug the solved $\Lambda_{1}, \Lambda_{2}$ and $\Lambda_{R}$ back into (37) to obtain the beamforming matrixes $\mathbf{W}_{1}, \mathbf{W}_{2}$ and $\mathbf{W}_{R}$.

Complexity Analysis: Note that the problem (45) can be effectively solved by three convex problems with scalar variables (51), (57), and (64). Based on the computational cost analysis in [18], the complexity of the lowcomplexity beamforming design for maximizing the sum retained energy is about $\mathcal{O}\left(M_{\text {Iter }} \cdot N^{3.5}\right)$. It is obvious that Algorithm 2 has lower computational complexity than Algorithm 1. Therefore, Algorithm 2 is more suitable for large-scale antennas system.

\section{Results and discussion}

In this section, some simulation results are provided to evaluate the effectiveness of the proposed schemes for UAV-enabled two-way relay system with SWIPT. As illustrated in Fig. 2, the UAV relay hovers at a constant altitude that is set as $d_{\nu}=5 \mathrm{~m}$ and the horizontal distance between UE 1 and UE 2 is fixed as $d_{h}=30 \mathrm{~m}$. Moreover, we denote the horizontal distances of UE 1-to-relay and relay-toUE 2 as $\xi d_{h}$ and $(1-\xi) d_{h}$, respectively. $\xi \in(0,1)$ is the location ratio of UAV relay. It is intuitive that the real distances of UE 1-to-relay and relay-to-UE 2 can be indicated as $L_{1}=\sqrt{d_{v}^{2}+\left(\xi d_{h}\right)^{2}}$ and $L_{2}=\sqrt{d_{v}^{2}+\left((1-\xi) d_{h}\right)^{2}}$, 


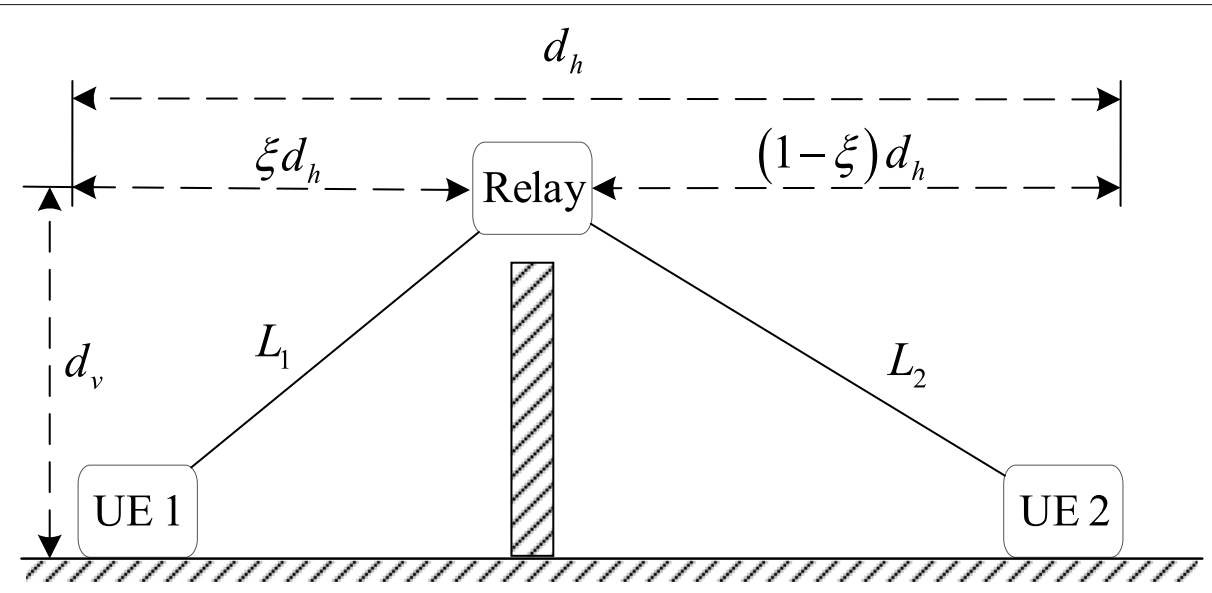

Fig. 2 The location of UAV-enable two-way relay system

respectively. Following [21, 30], we model the MIMO channel matrixes as $\mathbf{H}_{i}=\breve{\mathbf{H}}_{i} \sqrt{L_{i}^{-\varepsilon}}$ and $\mathbf{G}_{i}=\breve{\mathbf{G}}_{i} \sqrt{L_{i}^{-\varepsilon}}$, where $L_{i}^{-\varepsilon}$ strands for the large-scale fading, $\breve{\mathbf{H}}_{i}$ and $\breve{\mathbf{G}}_{i}$ indicate the small-scale Rician fading (Rician factor $K=$ 5). Similar to [17], the path loss exponent of the high altitude transmission is set as $\varepsilon=2$. For all simulations, we assume that the noise variances at all transceivers are same as $\sigma_{R}^{2}=\sigma_{i, z}^{2}=\sigma_{i}^{2}=-60 \mathrm{dBm}$. We also specify $\eta=0.8$, $\xi=0.5, N=2, T=1$ second, $\rho_{i}=0.5, \gamma_{i}=34 \mathrm{~dB}$, $E_{i}^{I N I}=50 \mathrm{~mJ}, P_{i, \operatorname{Max}}=20 \mathrm{dBm}$ and $P_{R, \text { Max }}=20 \mathrm{dBm}$ unless otherwise explained.
Figure 3 reveals the convergence performance of the proposed AO scheme and low-complexity GSVD scheme. It is found that the curve of the proposed AO scheme is similar to that of low-complexity GSVD scheme. They take no more than 5 iterations to converge. Compared with the AO scheme, the low-complexity GSVD scheme transforms the original optimization problem with matrix variables into the power allocation optimization problem with scalar variables. However, two schemes have a similar iteration process. Interestingly, the proposed $\mathrm{AO}$ scheme has a better performance of the sum retained energy than that of low-complexity GSVD scheme.

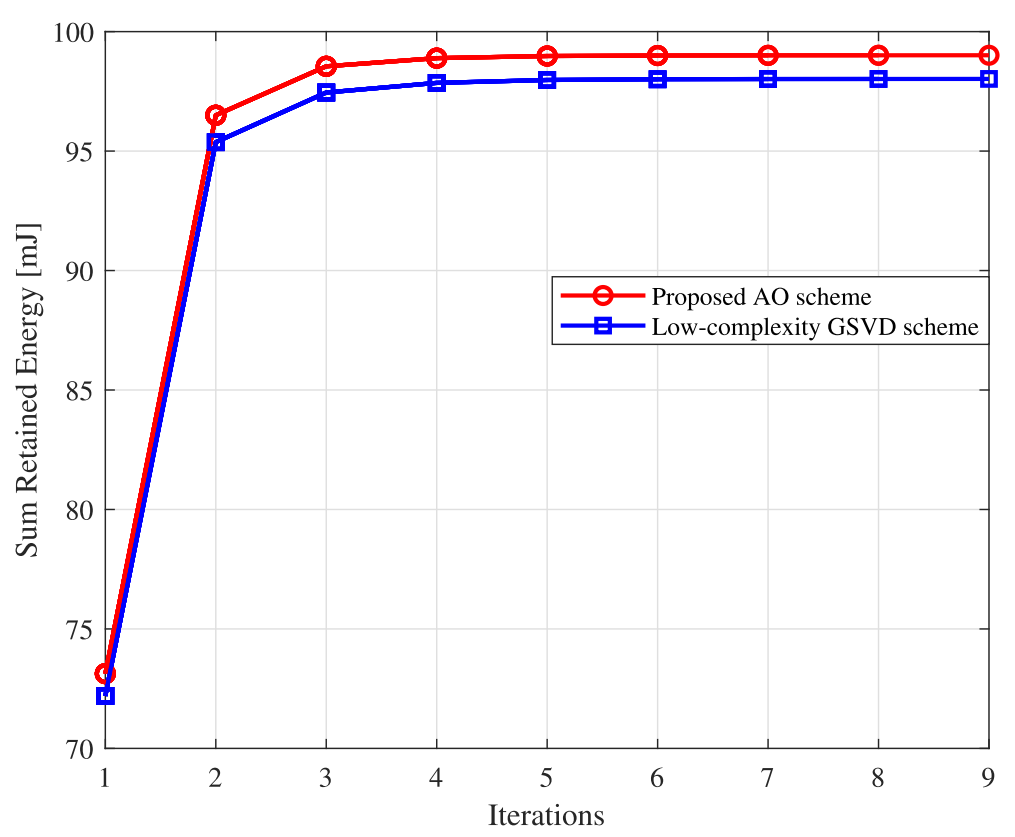

Fig. 3 Sum retained energy versus the number of iterations 

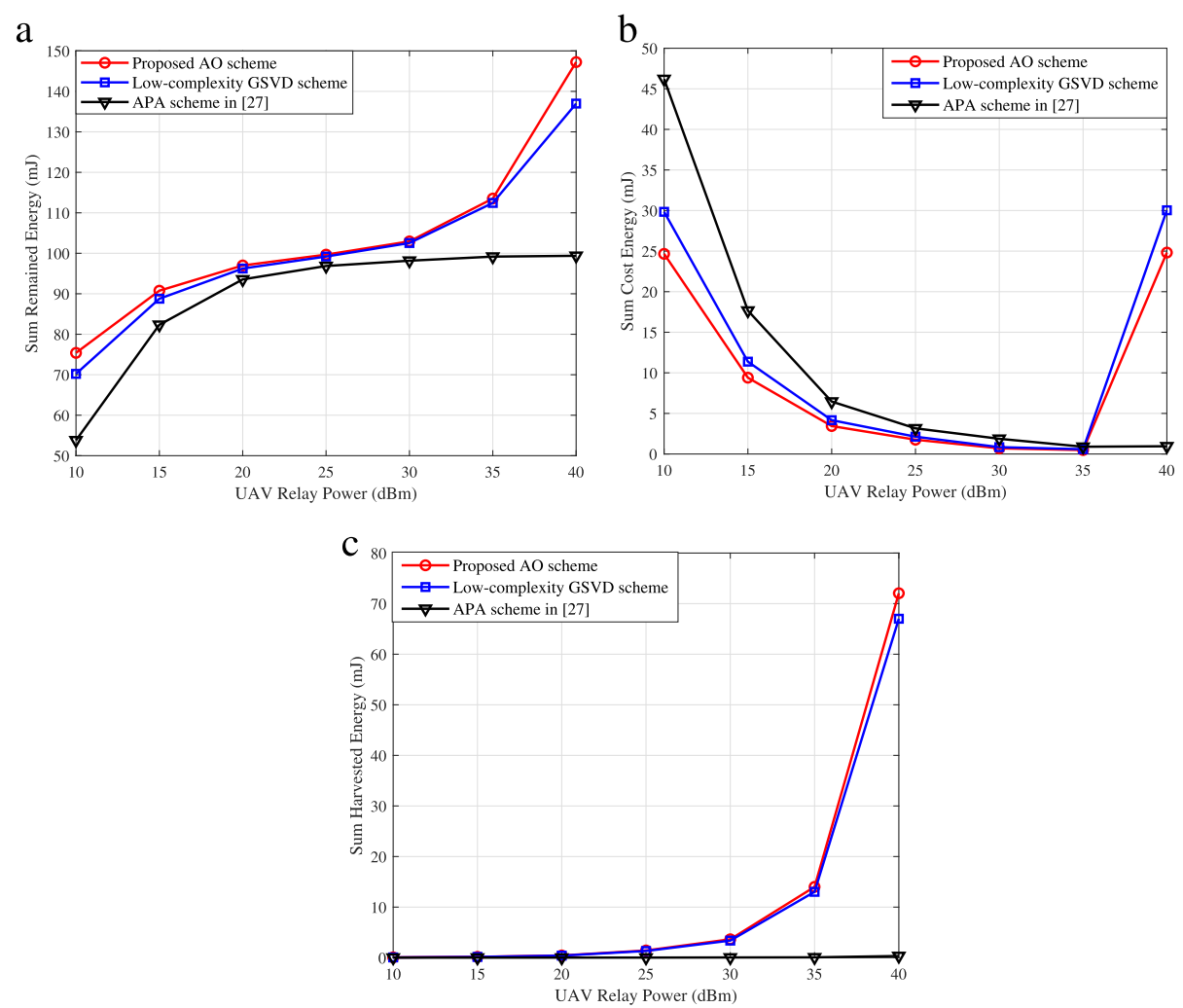

Fig. 4 Various performance results of different schemes versus the maximum transmission power of UAV relay (dBm) $P_{R, \text { Max }}$ a Sum remained energy versus $P_{R, \text { Max }}$; b Sum cost energy versus $P_{R, \text { Max }}$ c Sum harvested energy versus $P_{R, \text { Max }}$

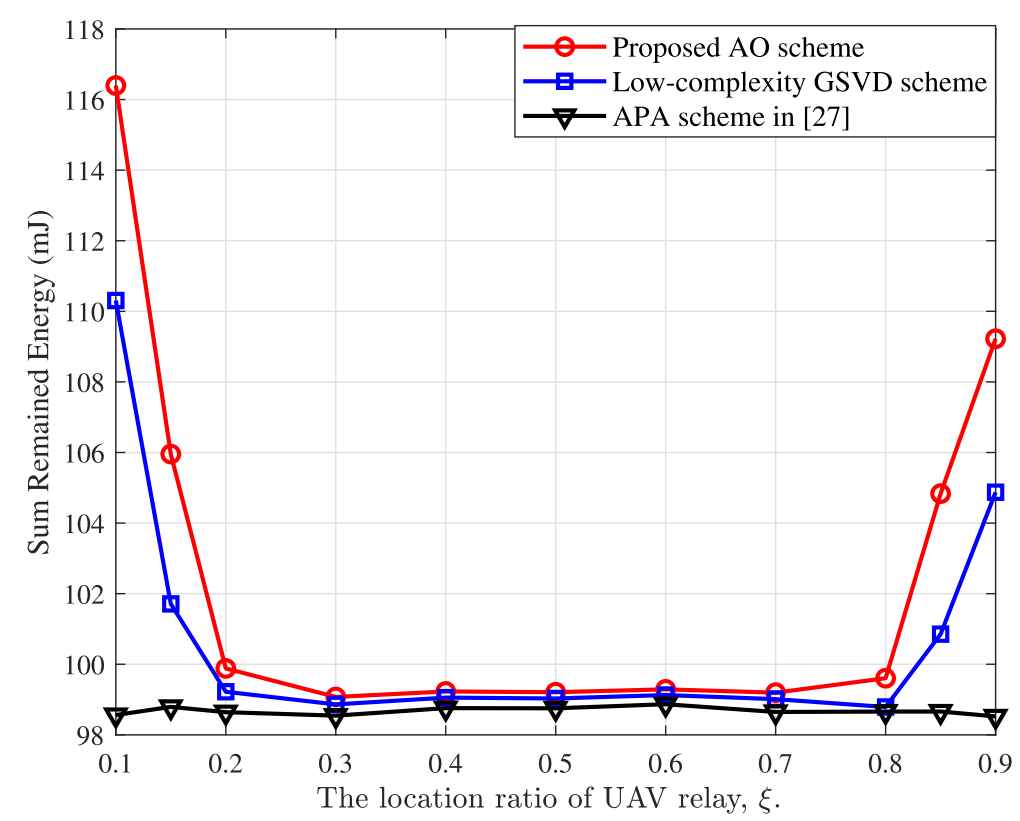

Fig. 5 Sum remained energy versus the location ratio of $U A \bigvee$ relay $\xi, P_{R, M a x}=25 \mathrm{dBm}$ 
Figure 4 depicts the various performance results of different schemes versus the maximum transmission power of UAV relay. It is intuitive from Fig. 4a that both the proposed AO scheme and low-complexity GSVD scheme significantly outperform the average power assignment (APA) scheme in [31]. This is because compared to APA scheme, the proposed $\mathrm{AO}$ scheme and low-complexity GSVD scheme can take full advantage of the robust beamforming matrixes and the effective PS factors to match the MIMO channels. Furthermore, the performance gaps between two proposed schemes and APA scheme grow as the maximum transmission power of UAV relay increases. Besides, it is found that the gap between the proposed AO scheme and low-complexity GSVD scheme is quite small due to the similar iterative process.

In Fig. 4b, we check the influence of the maximum transmission power of UAV relay on the sum cost energy of three schemes. It is seen that the APA scheme needs to cost more energy for the same SNR threshold than the proposed AO scheme and low-complexity GSVD scheme at low $P_{R, \operatorname{Max}}$. Moreover, as expected, the cost energy of APA scheme decreases as $P_{R, \text { Max }}$ increases. This is because for the fixed SNR requirement and given PS factors, APA scheme requires less transmission power of UE to guarantee the SNR threshold as $P_{R, \text { Max }}$ grows.

In Fig. $4 \mathrm{c}$, we investigate the impact of $P_{R, \operatorname{Max}}$ on the sum harvested energy of three different schemes. It is observed that the proposed AO scheme and lowcomplexity GSVD scheme harvest more energy than APA scheme at high $P_{R, \text { Max }}$. This happens because compared to APA scheme, two proposed schemes transfer more energy in MAC phase which results in the higher received power at UEs in BC phase. Besides, two proposed schemes have more efficient beamforming designs and more suitable PS factors to satisfy the SNR thresholds. Specifically, the performance gap of harvested energy between the proposed AO scheme and low-complexity GSVD scheme allocation factor algorithm is small due to the similar iteration procedure.

Figure 5 illustrates the influence of the location ratio of UAV relay $\xi$ on the sum remained energy of three schemes. Obviously, the proposed AO scheme and lowcomplexity GSVD scheme have better performance than APA scheme. In particular, the better remained energy performance of two proposed schemes are obtained when the UAV relay is closer to UEs. One reason is that the closer UAV relay is located to UEs, the more EH efficiency UEs have. In addition, when UAV relay is closer to UE, the MIMO channel between UAV relay and UE has better channel quality. This is consistent with the simulation result described in [18].

\section{Conclusion}

In this paper, the joint transceiver beamforming and PS factor optimization of UAV-enabled two-way relaying system have been investigated. SWIPT technique was considered at UEs to prolong the battery life. We proposed the AO scheme and the low-complexity GSVD scheme to maximize the sum remained energy of two UEs. It was found that both the proposed AO scheme and lowcomplexity GSVD scheme significantly outperform APA scheme with fixed PS factors. In particular, the lowcomplexity GSVD scheme provides an outstanding balance between complexity and performance. More importantly, it is verified that the low-complexity GSVD scheme performs close to the proposed AO scheme. Besides, we conclude that the location ratio of UAV relay has a huge influence on the sum remained energy.

\section{Abbreviations}

UAVs:Unmanned aerial vehicles; SWIPT:Simultaneous wireless information and power transfer; UE:User equipment; DF:Decode-and-forward; PS:Power splitting; TS:Time switching; MIMO:Multiple-input multiple-output;

GSVD:Generalized singular value decomposition; EH:Energy harvesting; ID:Information decoding; RF:Radio-frequency; NOMA: Non-orthogonal multiple access; CSI: Channel state information; MAC:Multiple access; BC:Broadcast; SDP:Semi-definite programming; AO:Alternating optimization

\section{Acknowledgements}

The authors would like to thank the Editor and anonymous reviewers for their helpful comments and suggestions in improving the quality of this paper. This work was supported by the National Natural Science Foundation of China under Grant 61671184 and 61901137 and in part by the Department of Product Development Unit 4G5G Ericsson (China) Communications Co., Ltd.

\section{Authors' contributions}

JW is the main writer of this paper and proposed the main idea. GW conceived and designed the experiments. GC and BL participated in the design of the study and performed the statistical analysis. RZ and RZ undertook revision works of the paper. All authors read and approved the final manuscript.

Funding

This work was supported by the National Natural Science Foundation of China under Grant 61671184 and 61901137 and in part by the Department of Product Development Unit 4G5G Ericsson (China) Communications Co., Ltd.

Availability of data and materials

Data sharing is not applicable to this article as no datasets were generated or analyzed during the current study.

\section{Competing interests}

The authors declare that they have no competing interests.

\section{Author details}

${ }^{1}$ Communication Research Center, Harbin Institute of Technology, 150001 Harbin, China. ${ }^{2}$ Ministerial Key Laboratory of JGMT, Nanjing University of Science and Technology, 210094 Nanjing, China.

Received: 17 June 2020 Accepted: 29 September 2020

Published online: 27 October 2020

\section{References}

1. G. Mu, Joint beamforming and power allocation for wireless powered UAV-assisted cooperative NOMA systems. EURASIP J. Wirel. Commun. Netw. 1, 1-14 (2020). https://doi.org/10.1007/13638.1687-1499 
2. N. Zhao, et al, UAV-assisted emergency networks in disasters. IEEE Wireless. Commun. 26(1), 45-51 (2019). https://doi.org/10.1109/MWC. 2018.1800160

3. Y. Yan, W. Shi, X. Zhang, Design of UAV wireless power transmission system based on coupling coil structure optimization. EURASIP J. Wirel. Commun. Netw. 67, 1-13 (2020). https://doi.org/10.1186/s13638-02001679-4

4. N. Zhao, et al, Joint trajectory and precoding optimization for UAV-assisted NOMA networks. IEEE Trans. Commun. 67(5), 3723-3735 (2019). https://doi.org/10.1109/TCOMM.2019.2895831

5. S. Jia, J. Zhang, H. Zhao, R. Zhang, Relay selection for improved security in cognitive relay networks with jamming. IEEE Wirel. Commun. Lett. 6(5), 662-665 (2017). https://doi.org/10.1109/LWC.2017.2728528

6. C. Li, S. Zhang, P. Liu, F. Sun, J.M. Cioffi, L. Yang, Overhearing protoco design exploiting intercell interference in cooperative green networks. IEEE Trans. Veh. Technol. 65(1), 441-446 (2016). https://doi.org/10.1109/ TVT.2015.2389826

7. Q. Song, F. Zheng, Y. Zeng, J. Zhang, Joint beamforming and power allocation for UAV-enabled full-duplex relay. IEEE Trans. Veh. Technol. 68(2), 1657-1671 (2019). https://doi.org/10.1109/TVT.2018.2889349

8. B. Ji, Y. Li, D. Cao, C. Li, S. Mumtaz, D. Wang, Secrecy performance analysis of UAV assisted relay transmission for cognitive network with energy harvesting. IEEE Trans. Veh. Technol. 69(7), 7404-7415 (2020). https://doi. org/10.1109/TVT.2020.2989297

9. X. Hu, K. Wong, Z. Z.heng. K. Yang and, UAV-assisted relaying and edge computing: scheduling and trajectory optimization. IEEE Trans. Wirel. Commun. 18(10), 4738-4752 (2019). https://doi.org/10.1109/TWC.2019. 2928539

10. L. Li, T. Chang, S. Cai, UAV positioning and power control for two-way wireless relaying. IEEE Trans. Wirel. Commun. 19(2), 1008-1024 (2020). https://doi.org/10.1109/TWC.2019.2950301

11. B. Li, J. Yang, H. Yang, G. Liu, R. Ma, X. Peng, Decode-and-forward cooperative transmission in wireless sensor networks based on physical-layer network coding. Wirel. Netw., 1-7 (2019). https://doi.org/ 10.1007/s11276-019-02092-6

12. C. Li, P. Liu, C. Zou, F. Sun, J.M. Cioffi, L. Yang, Spectral-efficient cellular communications with coexistent one- and two-hop transmissions. IEEE Trans. Veh. Technol. 65(8), 6765-6772 (2016). https://doi.org/10.1109/TVT. 2015.2472456

13. W. Lu, S. Hu, X. Liu, C. He, Y. Gong, Incentive mechanism based cooperative spectrum sharing for OFDM cognitive loT network. IEEE Trans. Netw. Sci. Eng. 7(2), 662-672 (2020). https://doi.org/10.1109/TNSE.2019.2917071

14. C. Li, F. Sun, J.M. Cioffi, L. Yang, Energy efficient MIMO relay transmissions via joint power allocations. IEEE Trans. Circ. Syst. II Exp. Briefs. 61(7), 531-535 (2014). https://doi.org/10.1109/TCSII.2014.2327317

15. Z. Ding, S.M. Perlaza, I. Esnaola, H.V. Poor, Power allocation strategies in energy harvesting wireless cooperative networks. IEEE Trans. Wirel. Commun. 13(2), 846-860 (2014). https://doi.org/10.1109/TWC.2013. 010213.130484

16. Y. Ma, H. Chen, Z. Lin, Y. Li, B. Vucetic, Distributed and optimal resource allocation for power beacon-assisted wireless-powered communications. IEEE Trans. Commun. 63(10), 3569-3583 (2015). https://doi.org/10.1109/ TCOMM.2015.2468215

17. Z. Wen, X. Liu, S. Zheng, W. Guo, Joint source and relay design for MIMO two-way relay networks with SWIPT. IEEE Trans. Veh. Technol. 67(1), 822-826 (2018). https://doi.org/10.1109/TVT.2017.2727061

18. X. Zhou, Q. Li, Energy efficiency for SWIPT in MIMO two-way amplify-and-forward relay networks. IEEE Trans. Veh. Technol. 67(6), 4910-4924 (2018). https://doi.org/10.1109/TVT.2018.2819682

19. W. Wang, R. Wang, H. Mehrpouyan, N. Zhao, G. Zhang, Beamforming for simultaneous wireless information and power transfer in two-way relay channels. IEEE Access. 5, 9235-9250 (2017). https://doi.org/10.1109/ ACCESS.2017.2701830

20. R. Zhang, C.K. Ho, MIMO broadcasting for simultaneous wireless information and power transfer. IEEE Trans. Wirel. Commun. 12(5), 1989-2001 (2013). https://doi.org/10.1109/TWC.2013.031813.120224

21. B. Li, Y. Rong, Joint transceiver optimization for wireless information and energy transfer in nonregenerative mimo relay systems. IEEE Trans. Veh. Technol. 67(9), 8348-8362 (2018). https://doi.org/10.1109/TVT.2018. 2846556
22. J. Wang, G. Wang, H. Yang, B. Li, T. Zhang, Joint transceiver optimization for multiuser multi-antenna relay systems with energy harvesting. IEEE Access. 7, 151156-151167 (2019). https://doi.org/10.1109/ACCESS.2019. 2947171

23. J. Rostampoor, S.M. Razavizadeh, I. Lee, Energy efficient precoding design for SWIPT in MIMO two-way relay networks. IEEE Trans. Veh. Technol. 66(9), 7888-7896 (2017). https://doi.org/10.1109/TVT.2017.2681942

24. W. Wang, et al, Joint precoding optimization for secure SWIPT in UAV-aided NOMA Networks. IEEE Trans. Commun. 68(8), 5028-5040 (2020). https://doi.org/10.1109/TCOMM.2020.2990994

25. S. Yin, Y. Zhao, L. Li, F.R. Yu, UAV-assisted cooperative communications with time-sharing information and power transfer. IEEE Trans. Veh. Technol. 69(2), 1554-1567 (2020). https://doi.org/10.1109/TVT.2019. 2956167

26. W. Y.ang. X. Sun, Secure communication in noma-assisted millimeter-wave SWIPT UAV networks. IEEE Internet Things J. 7(3), 1884-1897 (2020). https://doi.org/10.1109/JOT.2019.2957021

27. Q. Qi, X. Chen, D.W.K. Ng, Robust beamforming for NOMA-based cellular massive ioT with SWIPT. IEEE Trans. Signal Process. 68, 211-224 (2020). https://doi.org/10.1109/TSP.2019.2959246

28. R. Wang, M. Tao, Joint source and relay precoding designs for MIMO two-way relaying based on MSE criterion. IEEE Trans. Signal Process. 60(3), 1352-1365 (2012). https://doi.org/10.1109/TSP.2011.2178598

29. R. Mo, Y.H. Chew, MMSE-based joint source and relay precoding design for amplify-and-forward MIMO relay networks. IEEE Trans. Wirel. Commun. 8(9), 4668-4676 (2009). https://doi.org/10.1109/TWC.2009.081294

30. L. Sboui, H. Ghazzai, Z. Rezki, M. Alouini, Achievable rates of UAV-relayed cooperative cognitive radio MIMO systems. IEEE Access. 5, 5190-5204 (2017). https://doi.org/10.1109/ACCESS.2017.2695586

31. G. Chen, J. Wang, G. Wang, Y. Zou, D. Zhao, in 2019 IEEE 90 th Vehicular Technology Conference (VTC2019-Fall). Residual energy optimization for MIMO SWIPT two-way relaying system, (Honolulu, 2019), pp. 1-5. https:// doi.org/10.1109/NTCFall.2019.8891084

\section{Publisher's Note}

Springer Nature remains neutral with regard to jurisdictional claims in published maps and institutional affiliations.

\section{Submit your manuscript to a SpringerOpen ${ }^{\circ}$ journal and benefit from:}

- Convenient online submission

- Rigorous peer review

- Open access: articles freely available online

- High visibility within the field

- Retaining the copyright to your article

Submit your next manuscript at $>$ springeropen.com 